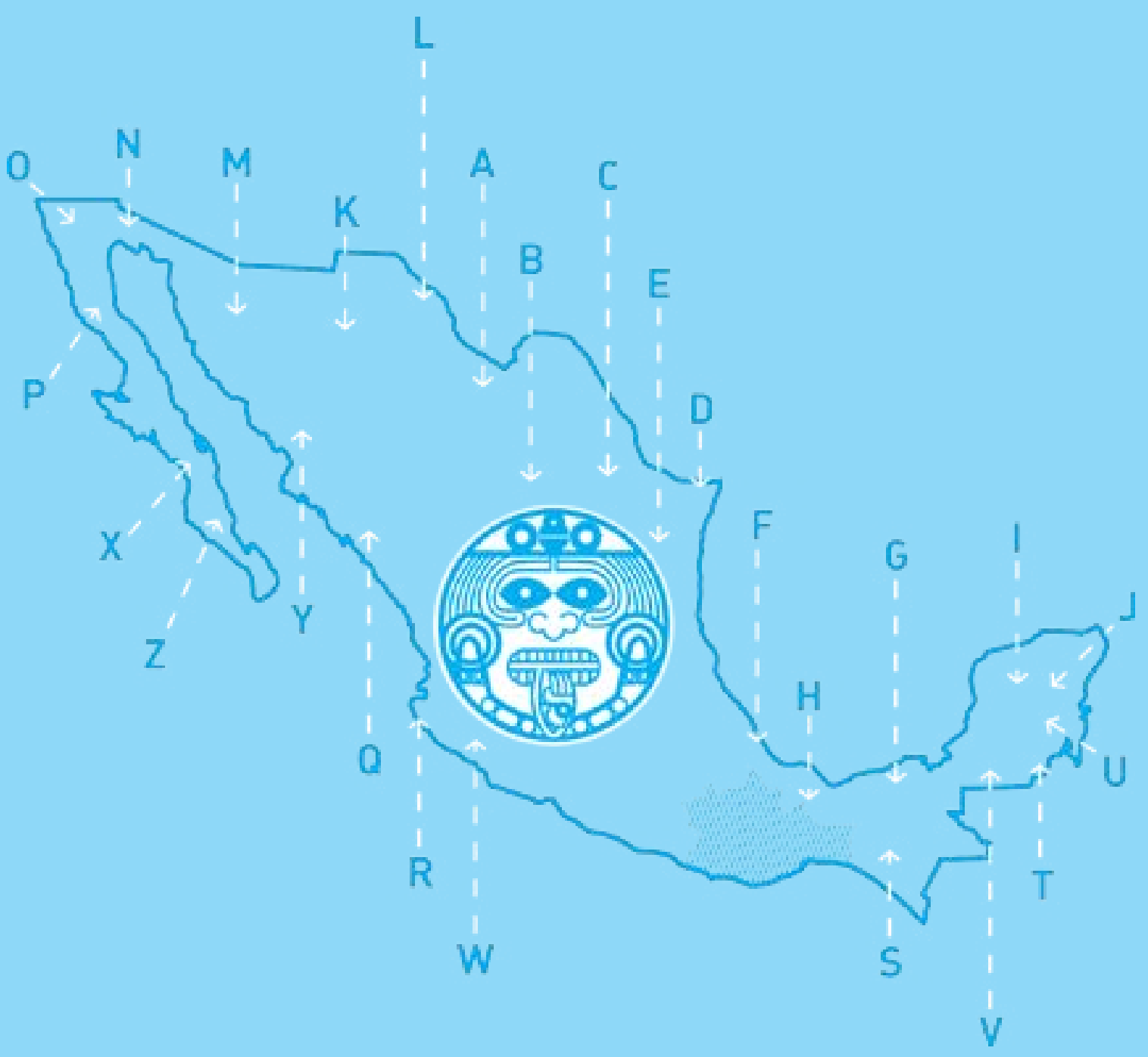

\title{
Algunas consideraciones sobre educación indígena y la formación de profesores indígenas en Oaxaca (México)
}

Some considerations for indigenous education and training of indigenous teachers in Oaxaca (México)

Algumas considerações sobre a educação e formação de professores indígenas em Oaxaca (México)

Bulmaro Vásquez Romero 


\section{Bulmaro Vásquez Romero ${ }^{1}$}

Magister en investigación educativa del Instituto Central de Ciencias Pedagógicas de La Habana, Cuba. Correo electrónico: bulmarovr@yahoo.com.mx

Fecha de recepción: 16 de febrero de 2015 / Fecha de aprobación: 3 de abril de 2015.

\section{Resumen}

La formación de profesores indígenas en el estado de Oaxaca es un problema a revisar en el marco de la historia de la educación indígena en México y en los desarrollos construidos en este campo en América Latina. Este análisis se enfoca en el caso de la Escuela Normal Bilingüe e Intercultural de Oaxaca, planteando la necesidad y la posibilidad de partir de la descolonización para acceder a la construcción de un proyecto de formación pedagógica que recupere el sentido político-cultural propio de la resistencia de los pueblos originarios de México.

\section{Palabras clave}

Colonización-descolonización, prácticas comunitarias, identidades indígenas, profesores indígenas, comunalidad.

\section{Summary}

Indigenous teachers' formation in the State of Oaxaca is a review question in the context of the history of Indian Education in Mexico, and constructed developments in this field in Latin America. This analysis focuses on the case of the Bilingual and Intercultural Normal School of Oaxaca, considering the necesity and possibility from decolonization to access the construction of a project of pedagogical training to recover the own cultural political sense of strenght of the original peoples of Mexico.

\section{Key words:}

Colonization-decolonization, community practices, indigenous identities, indigenous teachers, communality.

\section{Resumo}

A formação de professores indígenas no estado de Oaxaca é uma questão a ser revista no contexto da história da educação indígena no México e empreendimentos construídos neste campo na América Latina. Esta análise centra-se no caso da Escola Normal de Intercultural Bilíngüe Oaxaca, levantando a necessidade e possibilidade de descolonização para acessar a construção de um projeto de formação de professores para recuperar o próprio resistor sentido político -cultural dos povos nativos do México.

Palavras chave:

Colonização-descolonização, práticas comunitárias, identidades indígena, professores indígenas, comunalidade. 


\section{Introducción}

$\mathrm{L}$ a educación a través de las escuelas en comunidades indígenas de México representa en gran medida una acción colonizadora (Quijano, 2000) de parte del Estado y, por ende, ha estado lejos de responder a sus derechos culturales y lingüísticos. Aunque la legislación federal, particularmente la estatal, de los últimos veinte años reconoce en materia educativa el derecho de la población indígena a una educación en sus propias lenguas y basada en sus elementos culturales propios ${ }^{2}$, los hechos revelan que prevalecen prácticas educativas tendientes a la asimilación de estos pueblos y culturas; es decir, se trata de que por medio de la escuela la población indígena sea socializada para integrarse a la identidad nacional, olvidándose de sus orígenes, su historia, su lengua materna y, en suma, de su identidad como indígenas.

En el período histórico que emanó de la Revolución Mexicana de 1910, las políticas educativas pusieron énfasis en la castellanización y la alfabetización en español a nivel nacional; con ello, las lenguas indígenas fueron menospreciadas, sobre todo en las escuelas públicas, en las que se llegó a prohibir que los niños hablaran en su lengua materna. Socialmente se impuso la idea de la inferioridad de todo lo asociado a ser indígena (hablar lengua originaria, pensar el mundo desde su cosmovisión propia y, especialmente, practicar sus formas comunitarias de vida), frente a la supuesta superioridad del proyecto civilizatorio o modelo nacional.

Tan fuerte fue esa imposición, en la que la escuela de educación básica cumplió con su papel homogeneizador y enajenante de la cultura de los pueblos y comunidades indígenas, que desaparecieron muchas de las lenguas originarias de México, y en buena medida llegó hasta nuestros días, haciendo que muchos indígenas renieguen de su lengua y cultura propias. Se entiende por eso que:

Todo ese accidentado proceso implicó a largo plazo una colonización de las perspectivas cognitivas, de los modos de producir

2 A nivel federal, la Ley General de Educación establece el derecho de los pueblos indígenas a una educación basada en sus lenguas y culturas propias; esto se precisa más concretamente en la Ley General de los Derechos Lingüísticos de los Pueblos Indígenas de 2002. A nivel estatal se cuenta con la Ley Estatal de Educación (1995) y la Ley de Derechos de los Pueblos y Comunidades Indígenas del Estado de Oaxaca (1998), que en su conjunto contienen un marco jurídico a favor de la educación indígena tal y como sus pueblos y comunidades lo determinen. u otorgar sentido a los resultados de la experiencia material o intersubjetiva, del imaginario, del universo de relaciones intersubjetivas del mundo, de la cultura en suma (Quijano, 2000, p. 10).

Sin embargo, entre los propios indígenas hubo quienes se resistieron a esas prácticas de enajenación cultural y defendieron fuertemente lo propio a través de prácticas comunitarias de vida; gracias a ello, en pleno siglo XXI muchos pueblos y comunidades originarias de México se mantienen con vida y vienen haciendo planteamientos trascendentes, no solo para sí mismos en cuanto pueblos, sino de mucho valor para la vida del país.

En ese contexto cabe situar la experiencia de formación inicial de profesores indígenas bilingües e interculturales que, desde el año 2000, viene realizándose en varias escuelas normales públicas del país, con el fin de analizar y comprender mejor sus dinámicas internas, sus alcances y limitaciones. En estas notas examinamos con este fin el caso de la Escuela Normal Bilingüe e Intercultural de Oaxaca (ENBIO en adelante).

\section{Discusión}

Desde los años ochenta en Oaxaca (México), el magisterio mantiene una lucha sindical de resistencia frente a las políticas federales y estatales que atentan contra los trabajadores y contra la educación pública (Monsivais, et al., 1986; Kraemer, 2004; Martínez, 2005). Como parte de esta lucha, el magisterio indígena exigió durante años la creación de una escuela especialmente dedicada a formar nuevos profesores y profesoras indígenas, con preparación profesional adecuada para la defensa de las lenguas y culturas originarias de 16 pueblos aún vivos en el territorio de la entidad. Así surgió, en Febrero del año 2000, la ENBIO³.

De acuerdo con el plan de estudios que finalmente la SEP aprobó para este tipo de formación, a través de la Coordinación de Educación Intercultural Bilingüe y de la Dirección General de Educación Superior para Profesionales de la Educación ${ }^{4}$, se

3 El Decreto que la creó oficialmente fue firmado por el Gobernador del Estado de Oaxaca el 24 de Noviembre de 1999.

4 A causa de que crecieron en gran número las escuelas normales del país que acogieron el enfoque intercultural bilingüe para la formación de profesores de educación primaria, la SEP inició en 2004 y concluyó en 2008 la formulación del Plan 2004, específicamente para la licenciatura en educación primaria con enfoque intercultural bilingüe. Sin embargo, 
mantiene en lo fundamental la misma política nacional de formación para profesores de educación básica establecida desde el Plan de estudios 1997; solo se añade un línea de cursos dirigidos al aspecto lingüístico y cultural, y se hacen algunos cambios en los programas de los otros cursos, buscando favorecer una formación que considere, principalmente, la atención a la enseñanza de primeras y segundas lenguas.

Esto significa que a nivel curricular la propuesta pedagógica de formación no está centrada realmente en un modelo construído desde las prácticas culturales, lingüísticas y pedagógicas propias de los pueblos y comunidades indígenas (de Oaxaca, en este caso). Debido a esto, en la ENBIO se ha planteado la necesidad y la posibilidad de construir "desde dentro" algunas propuestas que permitan acercar mejor la formación inicial a una educación que reivindique las lenguas y culturas originarias, esto es, las identidades indígenas ${ }^{5}$.

Las discusiones académicas entre el profesorado y los estudiantes, sus búsquedas de propuestas didácticas y la implementación de otras prácticas formativas (además de las curriculares) a lo largo de quince años, nos ponen hoy frente a unas interrogantes que es necesario comenzar a responder, individual y colectivamente, para replantear, en lo posible, lo que hace la ENBIO en cuanto a la profesión de profesores indígenas. Estas interrogantes se refieren a:

- ¿Es posible formar profesores indígenas que construyan una educación y una pedagogía comunitaria e intercultural?

- ¿Cómo deconstruir la identidad cultural de subordinación o integración (a la identidad "nacional") para construir individual y colectivamente una identidad desde lo propiamente indígena?

- ¿Cómo avanzar de la descolonización a la construcción de una pedagogía cultural basada en la comunalidad?

dicho plan y sus programas de estudio mantienen en esencia la propuesta curricular nacional propia del Plan 97.

5 En el interés de hallar en la práctica un proyecto propio de formación de profesores indígenas oaxaqueños contemporáneos, se han desarrollado diversos seminarios, talleres y cursos entre profesores de la ENBIO, a veces con apoyo de expertos externos; también se han escrito diversas ponencias y análisis, como puede verse en la bibliografía de este documento.
Personalmente, considero que un proyecto de formación de profesores indígenas como el que se plantea la ENBIO, está enmarcado en la necesidad de descolonizarnos como profesores formadores mientras, al mismo tiempo, participamos de la formación de los nuevos profesores (nuestros estudiantes). Es decir, la propuesta misma de formación inicial en la escuela normal, ha de ser un proceso sostenido y de una participación muy clara y decidida para asumir: rupturas ideológicas y académicas con las prácticas de formación instituidas en las normales, sustituyéndolas con nuevas prácticas formativas basadas en el espíritu de la vida comunitaria que caracteriza a los pueblos indígenas que han resistido a la integración nacional (Maldonado, 2004; Chomsky, 2004).

Esto se da porque tanto los profesores como los estudiantes de la ENBIO estamos fuertemente imbuidos de los habitus de subordinación con que la sociedad nos formó a través de la escuela (Karabel y Hasley, 1976). Así, las expectativas y el interés con que se participa en la formación responden, en el fondo, a la reproducción de un modelo academicista y discursivo que solo superficialmente se identifica con la defensa de las lenguas y culturas indígenas. Dicho de otro modo, nuestras identidades, al llegar a la normal, están más del lado occidental que del lado indígena, y lo que hacemos en ella por nuestra formación no alcanza a dejar atrás esos habitus, poniendo en su lugar actitudes y prácticas pedagógicas (sobre todo) representativas de los modos indígenas aún vivos.

Considero que la formación de nuevos profesores indígenas para los pueblos originarios de Oaxaca y de México implica asumir un proceso de descolonización cultural en la escuela normal, más allá de solo abordar académicamente temáticas relativas a la defensa de nuestras lenguas y culturas originarias. Este proceso de descolonización puede acercar mucho mejor las prácticas de formación a la construcción de una educación y una pedagogía comunitaria e intercultural que de verdad defienda las identidades de los pueblos originarios (Verdín, 2008), es decir, nos acercará mucho más a prácticas basadas en la cosmovisión, la sabiduría y los principios de vida comunitarios propios de nuestros pueblos mesoamericanos ${ }^{6}$.

6 Floriberto Díaz, Juan José Rendón, Jaime Luna y Benjamín Maldonado aportan, a través de sus escritos, invaluables e imprescindibles elementos de conocimiento acerca de la comunalidad, desde los cuales hay que repensar la realidad de nuestros pueblos indígenas para fortalecer las propuestas a defender en distintos ámbitos de la vida socioeconómica, cultural y política. 
Solo asumiendo muy seriamente este proceso de deconstrucción de nuestras actuales identidades, los profesores formadores y los profesores en formación, en la ENBIO, estaremos trabajando individual y colectivamente para construir unas identidades desde lo culturalmente propio y, desde ahí, seremos capaces de plantearnos de otras maneras la interculturalidad (Ströbele-Gregor, Kaltmeier y Giebeler, 2010).

¿Cómo hacer esa deconstrucción de la identidad cultural? Creo que esto implica poner a las prácticas de re-conocimiento y de re-planteamiento como base de la nueva formación. Lo primero significa que estudiantes y profesores debemos trabajar preguntándonos por lo que la escuela nos enseñó a hacer, a sentir y a pensar. Es fundamental profundizar en el examen crítico de la socialización de que hemos sido objeto a través de la escolarización dominante, para darnos cuenta cabal de hasta dónde y con qué valores y actitudes se deformó nuestra identidad cultural como indígenas, y se nos formó para aceptar y reproducir la discriminación, la subordinación y la enajenación cultural en que vivimos.

A partir de reconocernos personalmente en ese proceso de formación escolarizada y social previa, revisando nuestras historias de vida personales y las de nuestros pueblos y culturas de origen, será posible que construyamos en la normal algunos replanteamientos básicos y potentes. Entre ellos, el que se refiere a la formación académica; es fundamental plantearse algo más que la formación tradicional, centrada en la reproducción de ciertos discursos, y buscar una formación cultural, ocupada de reivindicar, por medio de prácticas dentro y fuera de la escuela normal, las relaciones culturales propias de los pueblos indígenas oaxaqueños. Se trata, pues, de poner en el centro de la formación en la normal las prácticas culturales propias de nuestros pueblos y comunidades, con todo lo que esto significa como re-creación cultural de nosotros mismos.

Esto significa, también, que la formación ha de transitar el replanteamiento de la relación entre teoría y práctica. La nueva formación implica preguntarse continuamente por la relación entre saber y pensar, saber y hacer, saber y sentir, saber y ser, entre otras cosas que han de dar sentido a la acción, pensamiento y producción entre profesores y estudiantes.

A partir de estos reconocimientos y replanteamientos, creemos que es posible encausar la propuesta de formación cultural en la ENBIO, asumiéndola como la construcción individual y colectiva de una pedagogía cultural basada en la comunalidad (Chomsky y Meyer, 2004). Destacamos en este terreno las es- trategias y los principios orientadores que vemos fundamentales para hacer esto. A continuación se destacan las estrategias que hemos visualizado en el proyecto.

\section{Las prácticas como eje de la alternativa pedagógica}

A tono con lo que ha caracterizado al modo de resistencia de los pueblos indígenas, una formación cultural implica que la escuela normal ponga a sus propias prácticas en el centro de la propuesta. Es decir que la construcción individual y colectiva de una identidad basada en lo sustantivo de las culturas originarias, solo es posible por el establecimiento de nuevas prácticas de organización y participación en la vida dentro y fuera de la escuela normal.

La re-apropiación de los elementos culturales de los pueblos indígenas por profesores formadores y profesores en formación es una tarea que resuelve la práctica antes que la teoría; serán las prácticas de organización y de participación en las diversas tareas culturales las que iluminen o potencien la reflexión, el pensamiento propio de la propuesta de formación.

Desde nuestro punto de vista, entre tales prácticas se destacan: el tequio pedagógico y la investigación productiva. El primero, implica que el quehacer de profesores formadores y profesores en formación es un trabajo colectivo comprometido con la defensa de su escuela normal; una participación interesada y esforzada en mantener vivas las lenguas y culturas de nuestros pueblos. Para ello, el trabajo académico y cultural tiene que organizarse y desarrollarse en torno de eventos que se nutran de la participación de las etnias o pueblos presentes en la escuela normal, posibilitando la recreación de las lenguas y de los diversos elementos culturales sobresalientes (los más potentes para la identidad de cada cultura), y el encuentro entre pueblos, lenguas y culturas.

La investigación productiva es la práctica necesaria para que, a partir de una relación clara y comprometida de la escuela normal con las comunidades indígenas, interesadas en una nueva educación y en una nueva pedagogía comunitaria e intercultural, se avance en el diseño y experimentación de propuestas pedagógicas y didácticas. Este es el terreno más amplio y a la vez más complejo para que la formación pedagógico-cultural vaya encausándose y reencausándose, al mantener una participación comprometida de los profesores y de los estudiantes con sus comunidades y regiones de origen, en la búsqueda y construcción de propuestas que atiendan las necesidades lingüísticas y culturales más rele- 
vantes y apropiadas para reivindicar las identidades originarias y replantearlas en el marco de los avances científico-técnicos de nuestro tiempo.

En cuanto a los principios orientadores de la nueva formación de educadores indígenas oaxaqueños, cabe decir que son los ingredientes indispensables de la participación dentro y fuera de la escuela normal, tanto de los profesores formadores, como de los profesores en formación. Retomando a Chomsky, a Freire, Ipiña y a otros pensadores defensores de las luchas emancipatorias de nuestro tiempo a través de la educación, podemos señalar los siguientes principios.

\section{El compromiso con la integración, la independencia y la autodeterminación}

En este caso, la integración implica reconocerse como parte de una comunidad y trabajar por lo que la define en cuanto tal; significa que cada uno de los participantes en este proyecto de formación sabe que solo trabajando al lado de los demás, de los pares, de los iguales (en cuanto integrantes o pertenecientes a esta comunidad), se lograrán avances en la defensa de nuestras lenguas y culturas originarias. La razón de ser de cada uno está en pertenecer a esta comunidad y trabajar para hacerla cada día más digna y más fuerte. La integración también implica trabajar a favor de unirse con todas aquellas personas, comunidades, organizaciones sociales e instituciones que comparten la misma causa, en este caso, la defensa y revitalización de nuestras lenguas y culturas indígenas.

La independencia y la autodeterminación significan que el proyecto de formación, propio de la ENBIO, requiere de gran capacidad creativa para hallar los modos con los que, desde dentro, se construye una nueva pedagogía basada en lo comunitario, sin menoscabo de que se toma lo mejor de la propuesta curricular que permite el reconocimiento de estos estudios profesionales a nivel universitario.

\section{Por una comunidad abierta, tolerante y flexible}

Este principio es fundamental para no caer en los vicios de diversos autoritarismos con los que se reproducen la discriminación y la exclusión. Nos plantea el reto de establecer formas organizativas y de participación colectiva (comunitarias) poniendo en el centro el interés común, pero sin sacrificar, ni mucho menos someter o excluir, las expresiones diversas que pueden tener lugar como aportes individuales. Una colectividad es más grande y más creativa en la medida en que incluye la diversidad y sabe sacar provecho de ella para potenciar su identidad. La construcción de consensos permite precisamente reconocer y apreciar la diversidad alejándose de posiciones cerradas e inflexibles; esto, por supuesto, no es nada sencillo, pero es uno de los retos en que mejor se resuelve la creatividad individual y colectiva.

Además, este principio implica que la propuesta de formación cultural e intercultural se desarrolle en el marco de relaciones sociales amplias con organizaciones, comunidades e instituciones que hacen al movimiento indígena de nuestros días en el estado de Oaxaca, en el país y en otros países. Esto significa que no solo leamos a los intelectuales de este movimiento indígena (sobre todo el latinoamericano), sino que vayamos estableciendo relaciones de intercambio con universidades, organizaciones y pueblos que están avanzando en sus propuestas de educación propias, para aprender de ellos y con ellos. Igualmente, significa que hemos de construir relaciones fuertes de participación en proyectos propios de nuestras comunidades y pueblos indígenas de Oaxaca y de México, como medio para enriquecer y fortalecer nuestras propuestas en la normal.

\section{Capacidad de resistencia desde la creatividad}

Un proyecto como el que se pretende en la ENBIO pasa por la necesidad de vencer diversos retos; hay que reconocer que los obstáculos no solo son construcciones relacionadas con el estado y sus políticas, sino que algunas veces es desde dentro que se construyen y se hacen fuertes las trabas, las limitaciones. Entonces, se requiere de un permanente trabajo individual y colectivo que ponga mucha más fuerza en la creatividad, en el optimismo, en el entusiasmo de construir juntos lo que nuestros pueblos y comunidades aprecian más de sí mismos. Al participar de esta manera, cada uno tiene la oportunidad de hacer su propio crecimiento como persona integrada a su comunidad, a su cultura, a su pueblo. También será de esta manera como se disfrute la capacidad de encuentro con los otros, con los diferentes, con quienes compartimos la defensa de la vida comunitaria. 


\section{Conclusión}

Las posibilidades de construir un proyecto de formación de profesores indígenas que defiendan las identidades culturales propias de los pueblos originarios en Oaxaca, implican un proceso emancipatorio en que los sujetos de la formación recuperen el sentido y las prácticas comunitarias indígenas y las pongan en el centro de nuevas prácticas formativas. Este proceso demanda una participación comprometida y autocrítica de dichos sujetos en el movimiento indígena que, a nivel nacional e internacional, reivindica los derechos colectivos de los pueblos originarios. 


\section{Referencias}

Acevedo, S., et al. (2004). Tequio Pedagógico: colaboración pedagógica en comunidad. En Meyer, Lois y Maldonado, Benjamín. (Comp.). Entre la normatividad y la comunalidad. Experiencias educativas innovadoras del Oaxaca indígena actual. Oaxaca: Instituto Estatal de Educación Pública de Oaxaca, pp. 528-568.

Freire, P. (1997). Pedagogía de la autonomía. México: Siglo XXI.

Gabriel, F. (2009). La educación alternativa y el movimiento pedagógico en el discurso de los maestros democráticos de Oaxaca, como contexto de la educación bilingüe e intercultural (19892003). Ensayos sobre historia, política, educación y literatura de Oaxaca. Oaxaca: IIHUABJO, pp. 289-340.

García, E., et al. (2004). La escuela Normal Bilingüe e Intercultural de Oaxaca. En Meyer, Lois y Maldonado, Benjamín. (Comp.). Entre la normatividad y la comunalidad. Experiencias educativas innovadoras del Oaxaca indígena actual. Oaxaca: Instituto Estatal de Educación Pública de Oaxaca, pp. 476-510.

Karabel, J., y Halsey, H. (1976). Poder e ideología en educación. New York: Oxford Press.

Kraemer, G. (2004, Enero-Julio). Cultura política indígena y movimiento magisterial en Oaxaca. Alteridades, No. 27. México: Universidad Autónoma Metropolitana-Iztapalapa, pp. 135-146.

Maldonado, B. (2004). Comunalidad y educación en Oaxaca. En Meyer, Lois y Maldonado, Benjamín. (Comp.). Entre la normatividad y la comunalidad. Experiencias educativas innovadoras del Oaxaca indígena actual. Oaxaca: Instituto Estatal de Educación Pública de Oaxaca, pp. 24-42.

Maldonado, B. (2004). Resistencia y esperanza: el futuro de la comunalidad en un mundo globalizado. Entrevista a Noam Chomsky por Lois Meyer. En Meyer, Lois y Maldonado, Benjamín. (Comp.). Entre la normatividad y la comunalidad. Experiencias educativas innovadoras del Oaxaca indígena actual. Oaxaca: Instituto Estatal de Educación Pública de Oaxaca, pp. 613-636.

Martínez, V. (2005) ¡No que no, sí que sí!: Testimonios y crónicas del movimiento magisterial oaxaqueño. México: Sindicato Nacional de Trabajadores de la Educación, Sección 22.

Educación y CIUdAd No 29 Julio - Diciembre de 2015 ISSN 0123-0425 - PP 72 - 78
Monsivais, C., et al. (1986). Ataca Oaxaca. México: Leega.

Quijano, Aníbal. (2000). Colonialidad del poder, eurocentrismo y América Latina. Lima: Centro de Investigaciones Sociales (CIES).

Rendón, J. J. (2003). La comunalidad, modo de vida en los pueblos indios. Tomo I. México: Dirección General de Culturas Populares e Indígenas.

Reyes, S., y Vásquez, B. (2008). Formar en la diversidad: el caso de la Escuela Normal Bilingüe e Intercultural de Oaxaca (ENBIO). TRACE. Revista del Centro de Estudios Mexicanos y Centroamericanos. No. 053. México, pp. 83-99.

Rockwell, E. (2004). Herencias y contradicciones de la educación en las regiones indígenas. En Meyer, Lois y Maldonado, Benjamín. (Comp.). Entre la normatividad y la comunalidad. Experiencias educativas innovadoras del Oaxaca indígena actual. Oaxaca: Instituto Estatal de Educación Pública de Oaxaca, pp. 3-23.

Ströbele-Gregor, J., et al. (2009). Construyendo interculturalidad: Pueblos indígenas, educación y políticas de identidad en América Latina. Departamento Países Andinos y Paraguay. Unidad Coordinadora Pueblos Indígenas en América Latina y el Caribe.

Unda, M. P. (1998). Los maestros, las innovaciones y las redes en el espacio de lo no domesticado. Ponencia presentada en el Primer encuentro estatal de experiencias educativas innovadoras en educación básica y formación de docentes, organizado por el CEDES-22. Oaxaca, Febrero de 1998.

Unda, M. P. (2010). Procesos de subjetivación y construcción de lo colectivo, otros modos de formación. Ponencia presentada en el II Congreso Internacional sobre profesorado principiante e inserción profesional a la docencia, Buenos Aires, Argentina, 24 a 26 abril de 2010.

Verdín, Armando. (2008). P'ijyo’tan: pedagogías indias. San Cristóbal de las Casas, Chiapas: FONDEIB. 